\title{
SERotoninergic Modulation of Cortical And Respiratory Responses TO EPISODIC HyPOXIA
}

\author{
K. Budzinska \\ Department of Respiratory Research, Medical Research Center, Polish Academy of Sciences, Warsaw, Poland
}

\begin{abstract}
Biphasic respiratory response to hypoxia in anesthetized animals is accompanied by changes in the EEG mostly in the low EEG frequency bands. Serotonin is a potent modulator of cortical and respiratory activity through $5-\mathrm{HT}_{2}$ receptors. Present study investigated whether $5-\mathrm{HT}_{2}$ receptors might be involved in the EEG and respiratory relationship during normoxic and hypoxic respiration assessed from integrated phrenic (Phr) and hypoglossal (HG) nerve activities. EEG signal recorded from the frontal cortex was subjected to power spectral analysis in delta, theta, alpha, and beta frequency bands. Systemic administration of $5-\mathrm{HT}_{2}$ agonist DOI (1-(2,5-dimethoxy-4-iodophenyl)2 -aminopropane) enhanced tonic and lowered peak phasic respiratory activity, and increased frequency of bursts of Phr and HG activity. At the same time, EEG activity became desynchronized and arterial blood pressure (ABP) increased. Following DOI pretreatment, 11\% hypoxia induced an augmented respiratory response in comparison with the response in the baseline condition. ABP fell less then in the control hypoxia. EEG pattern changed less than in the baseline state. Subsequent administration of ketanserin, a 5$\mathrm{HT}_{2}$ antagonist increased respiratory activity, elicited a synchronization of EEG activity and hypotension. The respiratory response to hypoxia was attenuated and cortical response was more potent in comparison with that after DOI injection. Arterial blood pressure decreased more then during baseline hypoxic response. The results suggest that modulation of cortical synchronization and desynchronization through 5$\mathrm{HT}_{2}$ receptor active agents may impact to hypoxic respiratory response.
\end{abstract}

Key words: serotonin $5-\mathrm{HT}_{2}$ receptor, phrenic activity, hypoglossal activity, EEG, hypoxia

\section{INTRODUCTION}

Serotonin (5-HT) is present in the brainstem including the respiratory nuclei, phrenic and hypoglossal motoneurons [1]. Central respiratory activity is mostly mediated by $5 \mathrm{HT}_{1}$ and $5 \mathrm{HT}_{2}$ receptors. Moreover, serotonin drives directly hypoglossal motoneurons [2] and plays an important role in regulating hypoglossal motoneuron excitability via $5-\mathrm{HT}_{2 \mathrm{~A}}$ receptors. Although present in the carotid bodies [3], serotonin does not trigger the respiratory hypoxic response; however, at the level of the central respiratory system 5-HT influences dynamics of the respiratory response to hypoxia [4]. Brain serotoninergic system through $G$ protein-coupled receptors is an important state-dependent neuromodulator. Serotonin immunoreactive fibers and terminals are demonstrated in the sensory and motor cortex. There is a rich representation of 5$\mathrm{HT}_{2 \mathrm{~A}}$ receptors in several cortical areas in frontal, parietal, temporal and occipital lobes predominantly in the $\mathrm{Vth}$ cortical layer. The EEG signals origin mainly from postsynaptic potentials generated in this layer of the cortex [5]. Activation of serotoninergic receptors modulates cortical synapses [6] and the dynamics of cortical circuits [7]. Changes in the state of cortical activity influence respiratory activity during anesthesia and affect the respiratory response to hypoxia $[8,9]$. Previous study has revealed [10] that hypoxic respiratory response was associated with changes in the EEG activity. The effect was more prominent during hypoxic depression of respiration. Since phrenic and hypoglossal motoneuron and cortical output are under modulatory control of serotoninergic system through $5 \mathrm{HT}_{2}$ receptors, the present study was devoted to determine if serotonin $5-\mathrm{HT}_{2 \mathrm{~A}}$ receptors' activation would be effective in changing the association of the power spectral characteristics of EEG with the respiratory activity during eupneic ventilation and hypoxia. For this purpose an agonist of serotonin $5-\mathrm{HT}_{2 \mathrm{~A} / 2 \mathrm{C}}$ receptors 1-(2,5-dimethoxy-4-iodophenyl)-2-aminopropane (DOI) [11] was chosen, since its action upon cortical activity is documented [12] and, on the other hand, it also modulates respiratory activity [13]. To reverse DOI effects ketanserin, an antagonist of $5-\mathrm{HT}_{2 \mathrm{~A} / 2 \mathrm{C}}$ receptors [11] was used.

\section{Material AND METHODS}

\section{ANIMALS AND SuRgERY}

The study was approved by a Local Laboratory Animal Care and Use Committee. The experiments were performed on 11 adult male Wistar rats. Animals were anesthetized with $\alpha$-chloralose-urethane (75 and $800 \mathrm{mg} / \mathrm{kg}$, i.p., respectively). Following tracheal cannulation, the rats were paralyzed with pipecuronium bromide (Arduan, Gedeon-Richter, Budapest, Hungary) with an initial dose of $0.08 \mathrm{mg} / \mathrm{kg}$, repeated 
every hour, and were artificially ventilated. The vagus nerves were sectioned in the neck to eliminate the input from lung mechanoreceptors. The C5 phrenic nerve root (Phr) and hypoglossal nerve (HG) were cut at their distal ends and placed on bipolar silver electrodes. Stainless steel screws were positioned in the skull just above the durra (A 2.0, L 2.0). Reference electrode was placed in the frontal skull bone. Right femoral artery was cannulated to record arterial systolic and diastolic blood pressure (BP-2, Columbus Instruments, Columbus, Ohio, USA) and to measure of arterial blood gas content and $\mathrm{pH}$ (AVL Compact 2 Blood Gas Assembly, Roche Diagnostics, Graz, Austria). Right femoral vein was cannulated to inject drugs. Rectal temperature was maintained at $37-38^{\circ} \mathrm{C}$ by a heating pad.

\section{Neurophysiological RECORDINGS}

The activity of phrenic and hypoglossal nerve was amplified, and filtered $(5-2500 \mathrm{~Hz})$ with a NeuroLog system (Digitimer, Welwyn Garden, UK) and integrated with a time constant of $70 \mathrm{~ms}$. EEG potentials were filtered at $0.5-50 \mathrm{~Hz}$ band pass. Cortical and nerve signals were digitalized at the rate of $500 \mathrm{~Hz}$ and 5000 $\mathrm{Hz}$ respectively. Data of EEG, raw and integrated nerve signals and arterial blood pressure were captured, displayed and analyzed with Spike 2. v 6 data acquisition system (Cambridge Electronic Design Ltd, CED, Cambridge, UK).

\section{EXPERIMENTAL DESIGN}

Following the surgical procedure, a half an hour recovery period was maintained to stabilize records. The animals were ventilated with mild hyperoxic mixture to keep the oxygen pressure in arterial blood above 110 $\mathrm{mmHg}$. Such ventilation prevents chemoreceptor stimulation of respiration during control and after hypoxia. The level of blood gases and $\mathrm{pH}$ In each experiment the effects of hypoxic ventilation with $11 \%$ oxygen in nitrogen were tested three times: in baseline condition, 5 min after DOI and 5 min after ketanserin injection. Hypoxic exposures lasted about 1.5 minutes. DOI, 1(2,5-dimethoxy-4-iodophenyl)-2-aminopropane (SigmaAldrich, Poznan, Poland), a 5- $\mathrm{HT}_{2}$ agonist, was administered i.v. at a dose of $0.7 \mathrm{mg} / \mathrm{kg}$ half an hour after baseline hypoxia. About $20 \mathrm{~min}$ after completing hypoxia after DOI, ketanserin (Sigma-Aldrich, Poznan, Poland), a 5- $\mathrm{HT}_{2}$ antagonist, was given at a dose of 2 $\mathrm{mg} / \mathrm{kg}$. Before each treatment with DOI and ketanserin, arterial blood gases were checked and adjusted to baseline values when necessary. In separate 3 experiments, the effects of vehicle on the response to hypoxia were evaluated in the same time regimes.

\section{DATA ANALYSIS}

The EEG signal was divided into $5 \mathrm{~s}$ epochs and decomposed into four frequency bands: delta $(0.5-3.9$ $\mathrm{Hz})$; theta $(3.9-7.8 \mathrm{~Hz})$; and alpha $(7.8-13.7 \mathrm{~Hz})$; beta $(13.7-35.2 \mathrm{~Hz})$ in artifact free epochs. The power spectra within frequency bands were examined by means of a Fast Fourier Transform (FFT) analysis. Relative power for each frequency band was calculated as a percent of the control EEG power before each pharmacological treatment within the frequency range of 0.5-35.2 Hz. Absolute and relative EEG power in each band was averaged over periods of time corresponding to the baseline and the respiratory response to hypoxic exposure. Phrenic (A Phr) and hypoglossal nerve (A HG) amplitude, frequency of inspiratory bursts per minute (f) and minute activity (Axf Phr, Axf HG) were calculated and presented as a percent of control. The respiratory response to hypoxia was fractioned into four components: stimulation of breathing (HS), a decline of the response (HD) apnea (HA) and recovery (R). The EEG analysis was performed in the corresponding periods of time. Data were averaged in each fraction and presented as mean \pm SE. Alterations in respiratory and EEG activity during hypoxia in the control state and after DOI and ketanserin treatment were assessed with one-way ANOVA analysis. A P-value $<0.05$ was accepted as statistically significant.

\section{RESULTS}

\section{EFFECTS OF DOI AND KETANSERIN ON RESPIRATORY} ACtivity, EEG, AND Arterial Blood Pressure

Activation of $5 \mathrm{HT}_{2}$ receptors by systemic administration of DOI at a dose of $0.7 \mathrm{mg} / \mathrm{kg}$ depressed phasic phrenic and hypoglossal amplitude simultaneously in-

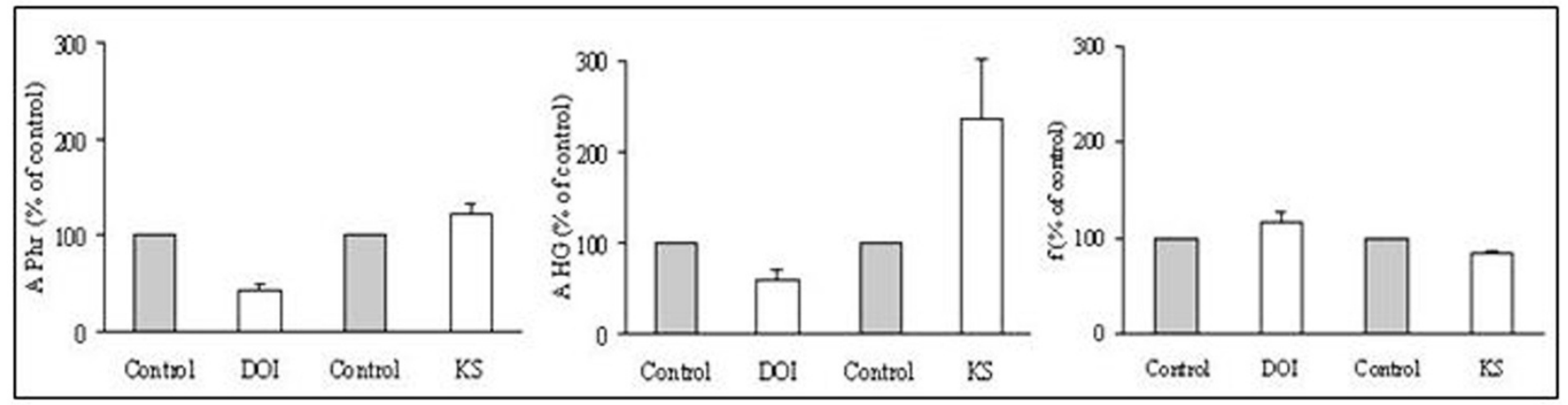

Fig. 1. Changes in peak amplitude of integrated activity of phrenic (A Phr) and hypoglossal (HG) nerve and respiratory frequency (f) evoked 5 min after systemic injection of DOI and ketanserin (KS). Effects were calculated as a percent of control before administration of agents. All effects were significant, $\mathrm{P}<0.05$. Values are means $\pm \mathrm{SE}, \mathrm{n}=8$. 


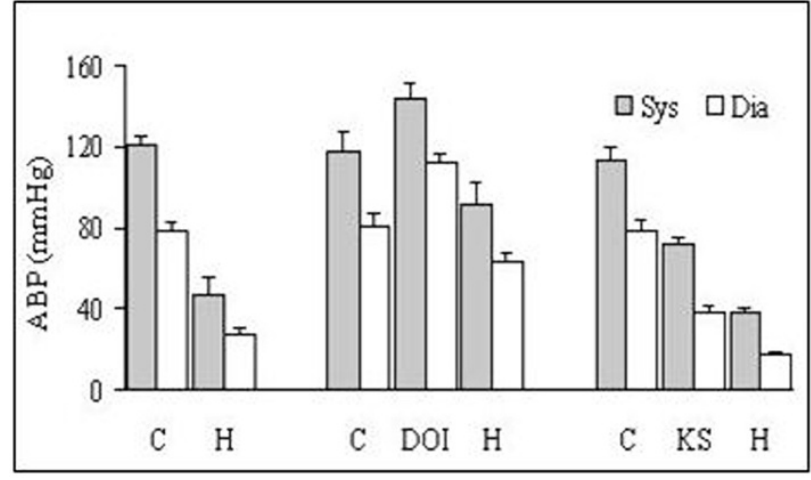

Fig. 2. Effect of DOI and ketanserin (KS) administration on systolic (Sys) and diastolic (Dia) arterial blood pressure (ABP) and the maximal response to hypoxia $(\mathrm{H})$ at baseline $(\mathrm{C})$, after DOI and ketanserin. All effects were significant, $\mathrm{P}<0.05$. Values are means $\pm S E, n=8$.

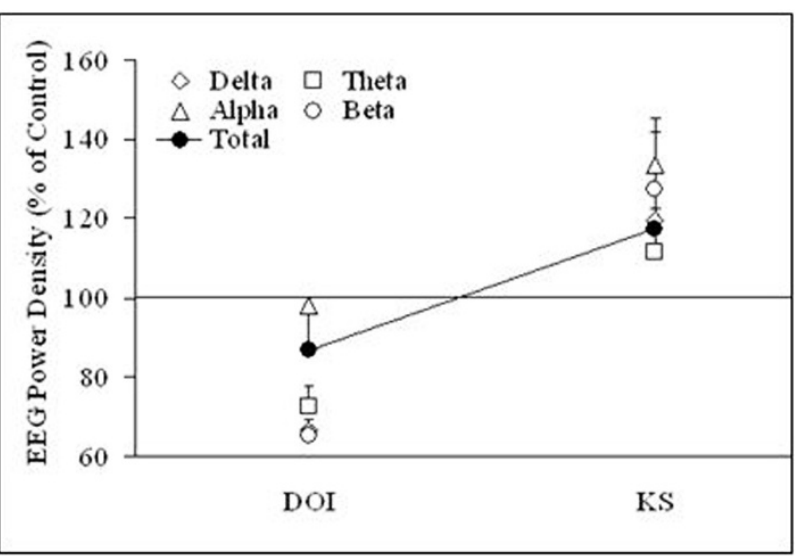

Fig. 3. Percent changes in power density of total EEG and delta, theta, beta, and alpha frequency bands elicited by DOI and ketanserin (KS). Horizontal line denotes the baseline values of power densities equal to $100 \%$. Values are means $\pm S E, n=8$. All effects were significant save alpha power density after DOI administration. creasing the frequency of respiratory bursts (Fig. 1). Low level tonic activity in hypoglossal nerve appeared, while in phrenic nerve tonic activity was not always present. DOI elicited an augmentation of both systolic and diastolic arterial blood pressure (Fig. 2) and attained its maximum before respiratory and cortical responses. All variables accomplished a stable level during 20 s. Systemic DOI attenuated significantly total EEG power density (Fig. 3) in comparison to the baseline EEG. A decrease of total EEG power density resulted from a decrease of all frequency bands, except for the alpha band that changed insignificantly.

Ketanserin administration at a dose $2 \mathrm{mg} / \mathrm{kg}$ elicited opposite effects to DOI. Inspiratory activity increased and frequency of bursts slowed down (Fig. 1). This effect was accompanied by a significant fall of ABP (Fig. 2). The amplitude of raw EEG signal and total power density of EEG was augmented (Fig. 3). The EEG activity attained a higher level than that in the baseline state. The power density in high, alpha, and beta frequency bands increased more than in the low, delta, and theta bands. One-way ANOVA showed a significant effect of DOI and ketanserin application on respiratory, EEG, and arterial blood pressure variables.

\section{Responses to Hypoxia FOLLOWING DOI AND KETANSERIN}

Figure 4 shows a hypoxic respiratory response in the baseline state and the effect of pretreatment with 5$\mathrm{HT}_{2}$ agonist and antagonist on this response. In the anesthetized and paralyzed animals, hypoxia introduced during eupneic ventilation evoked a typical increase of phrenic and hypoglossal activity corresponding to hyperpnea and a subsequent decline in response. In some experiments, hypoxic depression of inspiratory activity turned to apnea. In comparison with the phrenic hypoxic response, the biphasic hypoglossal response was more dynamic and attained higher values during stimulation of respiration and attenuated more than phrenic activity during a decline in response. Pharmacological treatment with $5-\mathrm{HT}_{2}$ agonist and antagonist modulated both phases of the hy-

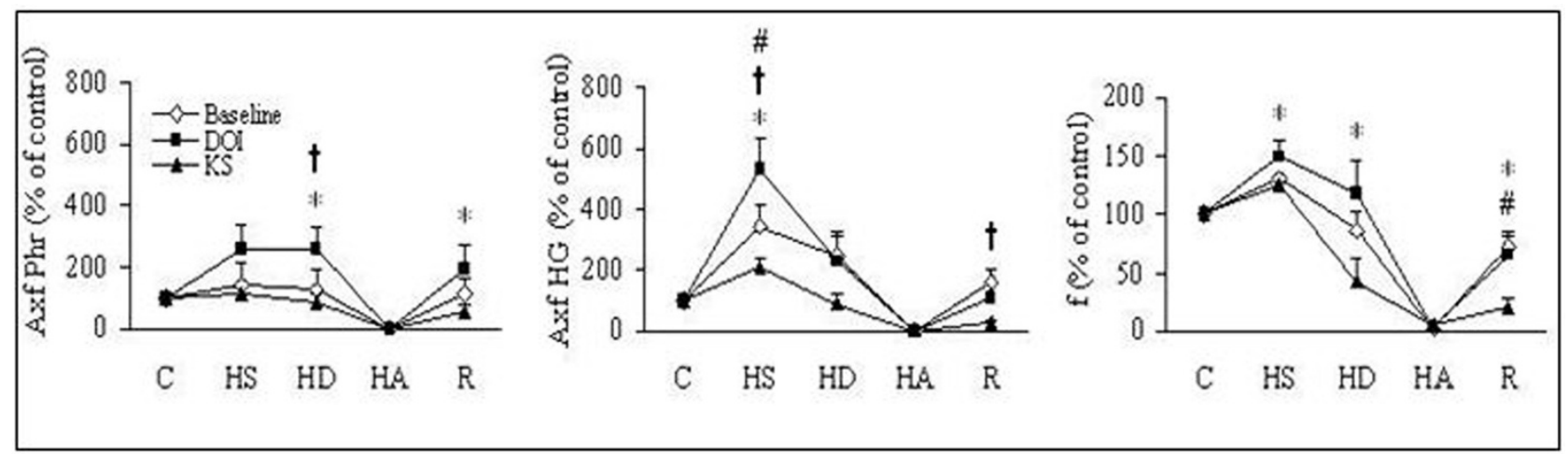

Fig. 4. Hypoxic effects on minute phrenic (Axf Phr) and hypoglossal activity (Axf HG) and respiratory frequency (f) divided into the phases of the response in baseline conditions and following DOI and ketanserin (KS) injection. Abbreviations: $\mathrm{C}$ - control value before induction of hypoxia, HS - hypoxic stimulation, HD - hypoxic decline, HA- hypoxic apnea, and R- recovery. Values are means $\pm S E, n=8$. *Significant difference between DOI and KS group; \#significant difference between Baseline and DOI group; †significant difference between Baseline and KS group; $\mathrm{P}<0.05$. 


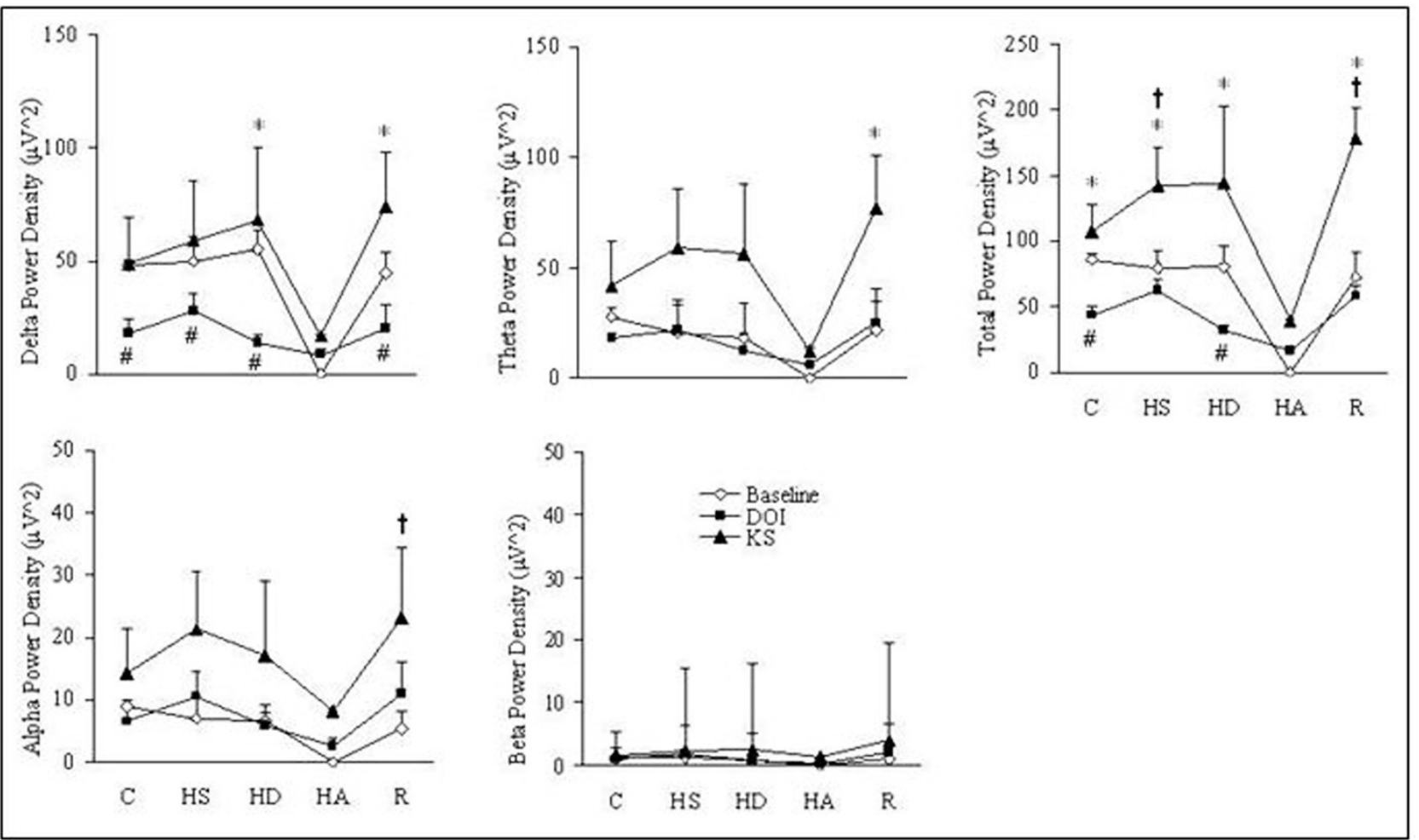

Fig. 5. Effects of hypoxia within the phases of the respiratory response on power density of total EEG and delta, theta, beta, and alpha frequency bands in baseline conditions and following DOI and ketanserin (KS) injection. Abbreviations same as in Fig 4. *Significant difference between DOI and KS group; \#significant difference between Baseline and DOI group; †significant difference between Baseline and KS group; $\mathrm{P}<0.05$.

poxic response by changing both magnitude and dynamics of the response.

After pretreatment with DOI, the respiratory hypoxic response was reinforced both in terms of initial stimulation and succeeding depression. Hypoxic effects in hypoglossal activity were more marked than those in phrenic activity. Subsequent ketanserin administration reversed the effect of DOI. Phrenic hypoxic response did not vary from the baseline response. The magnitude of hypoglossal response diminished below the baseline response. Respiratory frequency increased during stimulatory phase to a similar level as during baseline hypoxia, but it became strongly reduced during hypoxic decline.

In present study, a decline of the hypoxic response ended very often with apnea. It coincided with the lowest level of the arterial blood pressure in the course of hypoxia. Both, DOI and ketanserin affected arterial blood pressure (Fig. 3). A decreased of ABP elicited by hypoxia was smaller with DOI pretreatment and severe after ketanserin pretreatment. There was no consistent relationship between an appearance of apnea, used pharmacological agents and level of arterial blood pressure.

Fig. 5 presents effects of hypoxia on EEG power density spectra. Parallel to an augmentation of the hypoxic respiratory response after DOI, the EEG response to hypoxia flattened out in terms of absolute values. Alterations of EEG activity concerned the total EEG and delta power density, while the range of changes in the power density of theta, alpha, and beta rhythm correspond to those during baseline hypoxia. Only changes in the power density of delta frequency band and the power density of total EEG were statistically significant.

Ketanserin pretreatment stimulated the EEG response to hypoxia. The theta, alpha, and total EEG power density increased above the baseline hypoxic response; however, these effects were insignificant in comparison with the baseline response. Changes in delta power density were similar to that evoked during baseline hypoxia. During a decline of the hypoxic response and recovery from post-ketanserin hypoxia, changes in total EEG and delta power density were statistically different from those elicited by hypoxia after DOI pretreatment. When strong depression and apnea appeared, EEG activity and power density of all frequency bands became strongly suppressed or almost ceased. In the control and after ketanserin, the difference between the values of power density of all frequency bands during stimulation of respiration by hypoxia and apnea was much greater then that after DOI. There was a positive correlation between the level of hypoxic hypotension and a decrement of EEG power density for all frequency bands.

Administration of vehicle instead of DOI and ketanserin neither changed significantly the baseline activity of recorded parameters nor the hypoxic response. 


\section{DISCUSSION}

\section{Response to Systemic Administration of 5-HT 2} ReCEPTOR ACTIVE Agents

The study have shown that systemic administration of both agonist and antagonist of $5-\mathrm{HT}_{2}$ receptors caused parallel, prominent, and immediate changes in the phrenic and hypoglossal activity and EEG activity. Both respiratory and cortical effects of $5-\mathrm{HT}_{2 \mathrm{~A} / 2 \mathrm{C}}$ receptors agonist were reversed by $5-\mathrm{HT}_{2 \mathrm{~A} / 2 \mathrm{C}}$ antagonist.

The effects of systemic administration of DOI during eupneic ventilation consisted of diminution of phasic phrenic and hypoglossal nerve activity, an augmentation or appearance of tonic hypoglossal nerve activity and an increase in bursts frequency. In general, this respiratory response elicited in anesthetized, artificially ventilated animals corresponded to that evoked in the brainstem-spinal cord preparation $[13,14]$ and following DOI application on the ventral surface of the medulla [15]. Stimulatory effects of a $5-\mathrm{HT}_{2}$ agonist on respiratory activity concerned only the respiratory frequency in the present experimental conditions.

In the physiological state, serotonin increases EEG desynchronization and produces an increase in the vigilance level and motor activity by tonic activation of 5$\mathrm{HT}_{2}$ receptors [16]. Similarly to unanesthetized conditions, in anesthetized and paralyzed animals EEG reflects changes in the brain state. A decrease in total EEG power density and strong depression of delta power density elicited by $5-\mathrm{HT}_{2}$ receptor agonist DOI corresponded to desynchronization of EEG. Conversely, ketanserin increased EEG activity, thus it caused EEG synchronization. Similar effects were reported in anesthetized animals following ritanserin administration, another $5-\mathrm{HT}_{2}$ antagonist [16]. DOI-induced reduction in the power of EEG low frequency band oscillations was reversed by ketanserin. It is interesting that DOI did not change significantly the baseline level of alpha band activity, while ketanserin increased it mostly as it does when $5-\mathrm{HT}_{2}$ receptors are not activated earlier by an agonist [17].

Beside cortical and respiratory effects, a third prominent effect of systemic administration of $5-\mathrm{HT}_{2 \mathrm{~A} / 2 \mathrm{C}}$ receptors acting agents were changes in the arterial blood pressure; DOI increased while ketanserin decreased the arterial blood pressure. Ketanserin is not only a serotonin antagonist, but additionally an alpha1 adrenoreceptor antagonist [11] and a strong hypotension evoked by ketanserin might result from a blocking action of both types of receptors. DOI does not express alpha-1 adrenergic action. An analysis of EEG power spectra and arterial blood pressure changes in the time course of hypoxia showed a correlation between these two parameters. Such a correlation was present in all studied conditions. Whether the EEG changes evoked by serotonin active agents, DOI and ketanserin, during eupneic ventilation could, in part, reflect changes in arterial blood pressure is difficult to speculate. Hypotension alone induced, for instance, by hypovolemia, when blood gases remain constant, does not affect the EEG amplitude, until a fall of arterial blood pressure bellow $30 \mathrm{mmHg}$ [18].
DOI AND KETANSERIN EFFECTS On RESPONSES TO HYPOXIA

A previous study has demonstrated that the respiratory response to hypoxia was accompanied by changes in EEG activity [10]. The results of the present experiments show that modulation of serotoninergic neurotransmission by DOI and ketanserin altered the response profile to hypoxia of the phrenic and hypoglossal nerve activity. The time course of changes in the total power of EEG signal was related to that of the respiratory response to hypoxia. The EEG power density during the stimulatory phase of the hypoxic response altered slightly, but it became strongly depressed during apnea and then increased during recovery.

An increased hypoxic respiratory response occurred when exogenously activated $5-\mathrm{HT}_{2}$ receptors induced the EEG desynchronization. After DOI, when EEG activity was already desynchronized, the initial hypoxic response was associated with only slight EEG changes. Activation of EEG may facilitate a significant decrease in the delta band and total EEG power density that accompanied a decline of hypoxic response, suggesting some further increase of cortical desynchronization. Such an effect may be supportive in terms of mobilization of the system to improve general oxygenation. A depression of respiratory activity during hypoxia is of central origin [19]. This part of the hypoxic cortical response was modulated by 5 $\mathrm{HT}_{2}$ active agents. A probable contribution of cortical $5-\mathrm{HT}_{2}$ receptors to augmented hypoxic respiratory response following DOI may ensue from an increase in glutamate in the cortex, since systemic DOI increases glutamate concentrations in the ventral tegmental area [20] and cortical Fos expression [12].

The question arises of whether there is a common source of $5-\mathrm{HT}_{2}$ receptors involved in a relative interaction of cortical and respiratory output. Projections from serotoninergic raphe nuclei to cortical and respiratory structures originating from different parts of raphe nuclei do not designate these midline structures. A more probable site for initiating the serotonin-dependent relationship of cortical and respiratory activity seems the nucleus tractus solitarii (NTS). There are anatomical projections with reciprocal relation between the cortex and the NTS [21, 22] and it concerns also serotoninergic cells and terminals. During the respiratory response to hypoxia, information from the carotid body reaches the NTS, releasing glutamate which stimulates ventilation. A recent study has revealed that 5-HT released in the dorsomedial medulla during hypoxia acts on $5-\mathrm{HT}_{2}$ receptors and contributes to the initial hypoxic hyperventilation and subsequent respiratory decline [23].

Systemic application of $5-\mathrm{HT}_{2}$ receptor active agents evokes a generalized response in several systems in which $5-\mathrm{HT}_{2}$ receptors are present. It affects both central and peripheral mechanisms. The present results and hypothetical explanation suggest a necessity of further studies to differentiate between the peripheral and central serotonin $5-\mathrm{HT}_{2}$ receptors contributing to the relationship between cortical activity and responses to hypoxia. 
In conclusion, serotoninergic neurotransmission through $5-\mathrm{HT}_{2}$ receptors modulates the respiratory and EEG activity in eupneic breathing and during episodic hypoxia. Alterations in the cortical state through $5-\mathrm{HT}_{2}$ receptor active agents may influence the profile of the hypoxic respiratory response.

Acknowledgments: Supported by the statutory budget of the Medical Research Center, Polish Academy of Sciences and grant 2P05A 12229 from the Ministry of Education and Science in Poland. Renata Ilasz is acknowledged for cooperation during this study and Ewa Wielechowska for her excellent technical assistance.

Conflicts of interest: The author declared no conflicts of interest in relation to this article.

\section{REFERENCES}

[1] Okabe S, Mackiewicz M, Kubin L (1997) Serotonin receptor mRNA expression in the hypoglossal motor nucleus. Respir Physiol 110: 151-160.

[2] Berger AJ, Bayliss DA, Viana F (1992) Modulation of neonatal rat hypoglossal motoneuron excitability by serotonin. Neurosci Lett 143: 164-168.

[3] Gonzalez C, Dinger BG, Fidone SJ (1995) Mechanisms of carotid body chemoreception. In: Dempsey JA, Pack AI (eds.) Regulation of breathing. 79, pp. 391-471.

[4] Jacono FJ, Peng YJ, Kumar GK, Prabhakar NR (2005) Modulation of the hypoxic sensory response of the carotid body by 5 -hydroxytryptamine: role of the $5-\mathrm{HT}_{2}$ receptor. Respir Physiol Neurobiol 145: 135-142.

[5] Widman G, Schreiber T, Rehberg B, Hoeft A, Elger CE (2000). Quantification of depth of anesthesia by nonlinear time series analysis of brain electrical activity. Phys Rev E 62: 4898-4903.

[6] Zhou FM, Hablitz JJ. Activation of serotonin receptors modulates synaptic transmission in rat cerebral cortex. (1999) J Neurophysiol 82: 2989-2999.

[7] Zhang ZW, Arsenault D (2005) Gain modulation by serotonin in pyramidal neurones of the rat prefrontal cortex. J Physiol 566: 379-394.

[8] Boon JA, Garnett NB, Bentley JM, Milsom WK (2004) Respiratory chemoreflexes and effects of cortical activation state in urethane anesthetized rats. Respir Physiol Neurobiol 140: 243-256.

[9] Hamrahi H, Stephenson R, Mahamed S, Liao KS, Horner RL (2001) Selected Contribution: Regulation of sleepwake states in response to intermittent hypoxic stimuli applied only in sleep. J Appl Physiol 90: 2490-2501.

[10] Budzinska K, Ilasz R (2007) EEG and respiratory activity during acute intermittent hypoxia in anesthetized rats. J Physiol Pharm 57 Suppl 5: 55-62.

[11] Hoyer D, Hannon JP, Martin GR (2002) Molecular, pharmacological and functional diversity of 5-HT receptors. Pharm Bioch Behav 71: 533-554.

[12] Scruggs JL, Patel S, Bubser M, Deutch AY (2000) DOIInduced activation of the cortex: dependence on $5-\mathrm{HT}_{2 \mathrm{~A}}$ heteroceptors on thalamocortical glutamatergic neurons. J Neurosci 20: 8846-8852.
[13] Morin D, Monteau R, Hilaire G (1992) Compared effects of serotonin on cervical and hypoglossal inspiratory activities: an in vitro study in the newborn rat. J Physiol 451: 605-629.

[14] Al-Zubaidy ZA, Erickson RL, Greer JJ (1996) Serotonergic and noradrenergic effects on respiratory neural discharge in the medullary slice preparation of neonatal rats. Pflugers Arch 431: 942-949.

[15] King KA, Holtman JR Jr (1990) Characterization of the effects of activation of ventral medullary serotonin receptor subtypes on cardiovascular activity and respiratory motor outflow to the diaphragm and larynx. J Pharmacol Exp Ther 252: 665-674.

[16] Kantor S, Jakus R, Bodizs R, Halasz P, Bagdy G (2002) Acute and long-term effects of the $5-\mathrm{HT}_{2}$ receptor antagonist ritanserin on EEG power spectra, motor activity, and sleep: changes at the light-dark phase shift. Brain Res 943: 105-111.

[17] Moyanova S, Kortenska L, Kirov R (1998) High-voltage electroencephalogram spindles in rats, aging and $5-\mathrm{HT}_{2}$ antagonism. Brain Res 786: 55-63.

[18] Gavilanes AW, Vles JS, von Siebenthal K, Reulen JP, Nieman FH, van Sprundel R, Blanco CE (2001) Electrocortical brain activity, cerebral haemodynamics and oxygenation during progressive hypotension in newborn piglets. Clin Neurophysiol 112: 52-59.

[19] Vizek M, Pickett CK, Weil JV (1987) Biphasic ventilatory response of adult cats to sustained hypoxia has central origin. J Appl Physiol 63: 1658-1664.

[20] Martín-Ruiz R, Puig MV, Celada P, Shapiro DA, Roth BL, Mengod G, Artigas F(2001) Control of serotonergic function in medial prefrontal cortex by serotonin-2A receptors through a glutamate-dependent mechanism. J Neurosci 21: 9856-9866

[21] van der Kooy D, Koda LY, McGinty JF, Gerfen CR, Bloom FE.(1984) The organization of projections from the cortex, amygdala, and hypothalamus to the nucleus of the solitary tract in rat. J Comp Neurol 224: 1-24.

[22] Owens NC, Verberne AJ(1996) An electrophysiological study of the medial prefrontal cortical projection to the nucleus of the solitary tract in rat. Exp Brain Res 110: $55-$ 61.

[23] Kanamaru M, Homma I (2009) Dorsomedial medullary $5-\mathrm{HT}_{2}$ receptors mediate immediate onset of initial hyperventilation, airway dilation, and ventilatory decline during hypoxia in mice. Am J Physiol Regul Integr Comp Physiol 297: R34-41.

Author's address:

K. Budzinska

Department of Respiratory Research

Medical Research Center

Polish Academy of Sciences

5 Pawinskiego St.

02-106 Warsaw, Poland

Phone +48 22608525

Fax: +48226086622

kbudzinska@cmdik.pan.pl 